\title{
La valoración docente como mecanismo de mejoramiento del proceso de enseñanza-aprendizaje en la carrera de Administración de Empresas
}

\section{Teacher assessment as a mechanism for improving the teaching-learning processing in the career of business administration}

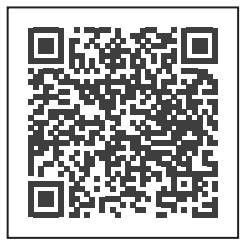

Palabras clave: educación superior, mejora continua hacia una formación integral, tasación formativa, valoración, Bolivia

Articulo de investigación:

Fecha de recepción:

10/10/2020

Fecha de aceptación:

09/12/2020

Esta publicación se encuentra bajo licencia:

Creative Commons

Reconocimiento-

NoComercial-

SinObraDerivada 4.0

Internacional

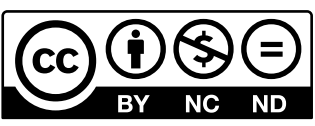

Said Rodrigo Morales Gamarra’

\section{Resumen}

La presente investigación enfatiza en la importancia de la tasación del desempeño docente en el proceso de formación, que permite describir las características desde una perspectiva del proceso enseñanza-aprendizaje, ya que la problemática hace mención de que no se conocen de manera objetiva y pertinente los aspectos inherentes a la labor formativa, lo que limita a la retroalimentación oportuna de la mejora continua en el proceso formativo. Es por esta razón por lo que se transversaliza la función formativa desde un enfoque integral docente-estudiante centrado en el problema de cómo evaluar el proceso de enseñanza universitaria, que pueda sustentar el perfeccionamiento del desempeño docente. El estudio tiene como objetivo describir la tasación docente dentro de la carrera de Administración perteneciente a la facultad de Ciencias Económicas y Financieras de la UAJMS, ubicada en la provincia Cercado del estado plurinacional de Bolivia.

Doctorante en Ciencias e Investigación, magíster en Ingeniería Financiera. Especialidad en educación superior. Docente, Facultad de Ciencias Económicas Financieras, Universidad Autónoma Juan Misael Saracho, Zona el Tejar Campus Universitario, Tarija, Bolivia. saidrodrigogamarra2@gmail.com, ORCID: https:// orcid.org/0000-0002-6411-7553 


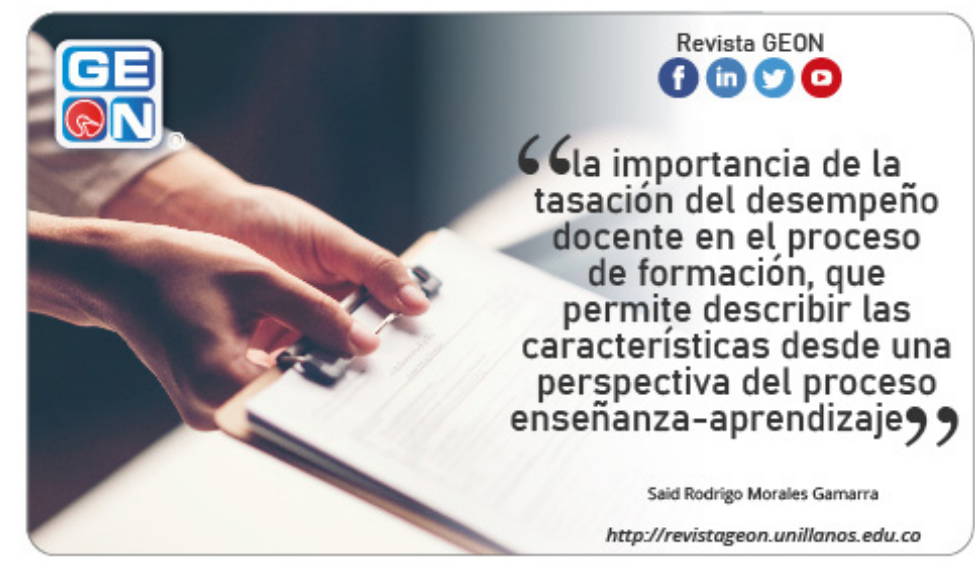

Cómo citar este artículo / Toreference this article:

Morales Gamarra, S. R. (2021). La valoración docente como mecanismo de mejoramiento del proceso de enseñanza-aprendizaje en la carrera de Administración de Empresas. Revista GEON (Gestión, Organizaciones Y Negocios), 8(1), e-271. https://doi. org/10.22579/23463910.271
El tipo de investigación utilizada fue descriptiva y se estudiaron los aspectos inherentes al proceso de formación. Se trabajó con 460 estudiantes como referencia poblacional y 20 estudiantes como muestra aleatoria, a los que se les realizó un cuestionario que hizo énfasis en los componentes del proceso de enseñanza. Luego se presentaron los resultados primarios, que permitieron llevar a cabo la discusión de los componentes observados en el estudio, como ser la organización y planificación de la labor formativa, la utilización de recursos tecnológicos y la formación integral de los futuros profesionales. Finalmente, se presentaron las conclusiones más relevantes del estudio, en las cuales se puede mencionar que la planificación y la organización de la cátedra en las diferentes asignaturas se realizó con base en el programa docente presentado. Se aplicaron sistemas de evaluación clásicos, que carecen de aspectos de retroalimentación, lo que limitó las instrucciones académicas respectivas, para lo cual también se utilizaron datos de estudios previos, que son parte de una investigación complementaria.

Palabras clave: educación superior, mejora continua hacia una formación integral, tasación formativa, valoración, Bolivia

Códigos JEL: M1. Administración, Administración de empresas, Administración de empresas, Gestión, Personal. 


\section{Abstract}

This research emphasizes the importance of assessing teacher performance in the training process, allowing the characteristics to be described from a perspective of the teaching-learning process, since the problem mentions that the aspects inherent to the teaching are not objectively and pertinently known. Formative work, which limits to a timely feedback of continuous improvement in the training process, is for this reason that the training function is mainstreamed from a comprehensive student teaching approach focused on the problem of how to evaluate the university teaching process that can support perfecting the teaching performance. The study aims to describe the teaching appraisal within the administration career belonging to the faculty of economic and financial sciences of the UAJMS, located in the Cercado province of the plurinational state of Bolivia.

The type of research used was descriptive, studying the aspects inherent to the training process. It was worked with 460 students as a population reference and 20 students as a random sample to whom a questionnaire was assigned emphasizing the components of the teaching process. Then, the primary results that allowed the discussion of the components observed in the study, such as the organization and planning of the training work, as well as the use of technological resources and the comprehensive training of future professionals, were presented. Finally, we presented the most relevant conclusions of the study, in which it can be mentioned that the planning and organization of the chair in the different subjects are carried out based on

the teaching program presented; classic evaluation systems are applied lacking feedback aspects, limiting the respective academic instructions for which also data from previous studies that are part of a complementary investigation were also used.

Keywords: Continuous improvement towards a comprehensive training; Formative appraisal; Higher education, Valuation; Bolivia 


\section{Introducción}

No cabe duda de la importancia de la educación superior y cómo esta contribuye a la formación pertinente de futuros administradores de empresas, que respondan al contexto social y a la solución de los problemas de su región. El presente estudio hace referencia a la problemática de que no se conocen de manera objetiva y pertinente los aspectos inherentes a la labor formativa y cómo poder evaluar el proceso de enseñanza universitaria en el centro de formación superior. El presente artículo científico responde a una estructura compuesta por un breve resumen de los aspectos centrales inherentes al estudio, así como a una introducción que considera la fundamentación y problemática como el contexto teórico, continúa con los resultados abordados y la discusión, resaltando los aspectos más importantes de las variables analizadas, y finaliza con las conclusiones.

\section{Contexto teórico}

La docencia desempeña un papel fundamental en el proceso formativo del educando en cuanto a los conocimientos, las habilidades, los valores y las actitudes (Gilio \& Ibarra, 2005).

De esta manera, se busca una intervención por parte del docente y estudiante y que cada uno asuma su rol y lo interprete de la mejor manera (Casanova, 2012).

El trabajo describe los componentes formativos, como ser la organización y la planificación de la cátedra, la or- ganización de esta, la evaluación del aprendizaje, la responsabilidad, la interacción y la motivación con los estudiantes. Estos componentes son parte fundamental del proceso de enseñanza-aprendizaje, la principal variable del estudio; también fueron desagregados en actividades operativas que son trabajadas en el aula y que responden a un diseño y un plan curricular.

La educación superior incorpora la informática y las comunicaciones, que permiten interactuar dentro de la actividad académica como recursos disponibles hacia una educación más inclusiva (González et al., 2017).

\section{Materiales y métodos}

De acuerdo con el objetivo de la investigación, su estructura, procedimientos y estrategias metodológicas aplicadas al presente trabajo, se trabajó a través de una guía de investigación, donde se incluyó una encuesta aplicada a los estudiantes.

Para cumplir este propósito, se consideró la investigación descriptiva, que permitió determinar con mayor precisión el objeto de estudio y su justificación, con lo que se determinó un diseño muestral que facilitó el trabajo con datos recolectados.

Se trabajó con un nivel de confianza del 95\%, para lo cual se aplicó un cuestionario a los estudiantes de la carrera de Administración de Empresas correspondiente a la facultad de Ciencias Económicas y Financieras. 
Dicha organización en los métodos de trabajo responde a un diseño muestral y a la utilización de instrumentos como el cuestionario. Esta herramienta fue el recurso relevante en el levantamiento de los datos obtenidos por parte de los estudiantes, fuente principal de insumos para poder analizar los resultados.

\section{Resultados}

De acuerdo con los datos presentados en este estudio, en la tabla 1 se muestra el número de ítems utilizado en el presente trabajo de investigación para analizar y describir las asignaturas referenciales desarrolladas por los docentes en función de las alternativas de respuestas utilizadas en el cuestionario, señalando los datos respectivos.

Según datos sistematizados, más del $60 \%$ de los encuestados mencionan que, con frecuencia, el docente facilita, al inicio de la clase, el contenido que será avanzado, lo que permite deducir que hubo una planificación y preparación de la clase de manera anticipada. El porcentaje restante
(40\%) sostuvo que no siempre algunos docentes explican y retroalimentan lo que avanzarán en clase; esto permite concluir que se debe trabajar más este aspecto dentro del aula.

Figura 1. Aspecto de contenido. Fuente: resultados a partir de las encuestas obtenidas de los estudiantes, año 2019.

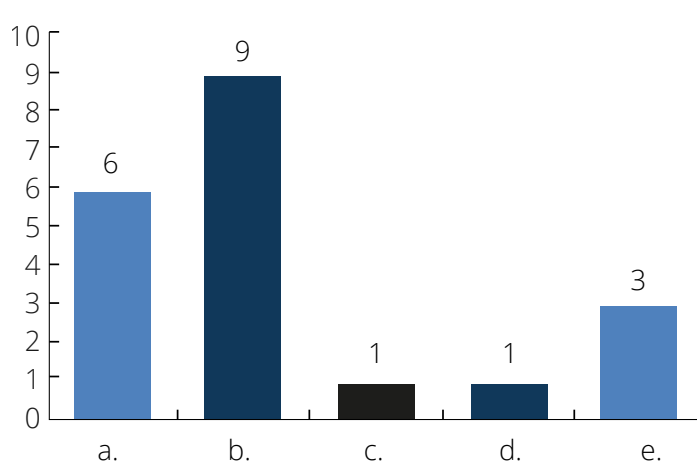

a. Siempre b. Frecuentemente c. A veces d. Casi nunca e. Nunca

De acuerdo con los resultados obtenidos, la mayor parte de los estudiantes indica que su docente frecuentemente realiza un análisis de los temas que expuso, lo que permite deducir que el docente considera la retroalimentación de los contenidos de avance un aspecto importante. El resto de los docentes no lo realizan permanente-

Tabla 1. Asignaturas correspondientes al semestre observado - 2019

\begin{tabular}{l|c|c|c}
\multicolumn{1}{c|}{$\begin{array}{c}\text { Materia } \\
\text { (Con mayor presencia) }\end{array}$} & \multicolumn{1}{c|}{$\begin{array}{c}\text { Alternativa de } \\
\text { respuestas }\end{array}$} & $\begin{array}{c}\text { Valor absoluto } \\
\text { (estudiantes) }\end{array}$ & Porcentaje (\%) \\
$\begin{array}{l}\text { Gestión Pública I } \\
\text { Estadística II }\end{array}$ & Siempre & 6 & 30 \\
\hline Gestión de Recursos Humanos II & Frecuentemente & 9 & 45 \\
\hline Marketing II & A veces & 1 & 5 \\
\hline & Casi nunca & 1 & 5 \\
\hline Gestión de Costos II & Nunca & 3 & 15 \\
\hline Total & & $\mathbf{2 0}$ & $\mathbf{1 0 0}$ \\
\hline Estudio de evaluación desempeño docente. Año 2019 & &
\end{tabular}


mente, un factor que tendría que mejorarse para un buen desempeño.

Figura 2. Análisis de las unidades temáticas en avance. Fuente: resultados a partir de las encuestas obtenidas de los estudiantes, año 2019.

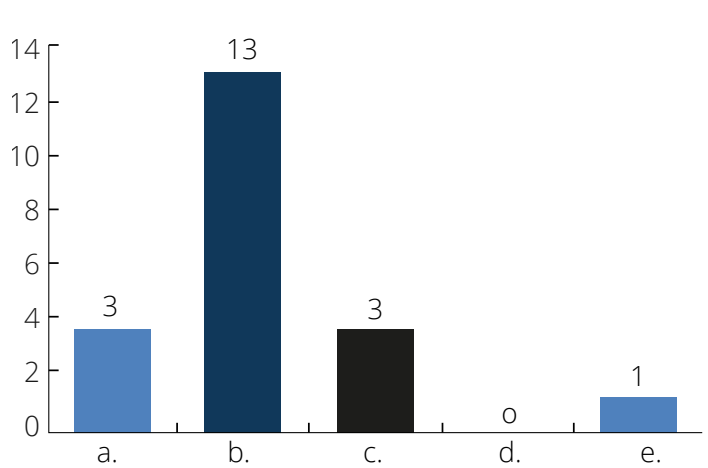

a. Siempre b. Frecuentemente c. A veces d. Casi nunca e. Nunca

Figura 3. Plan de la asignatura. Fuente: resultados a partir de las encuestas obtenidas de los estudiantes, año 2019.

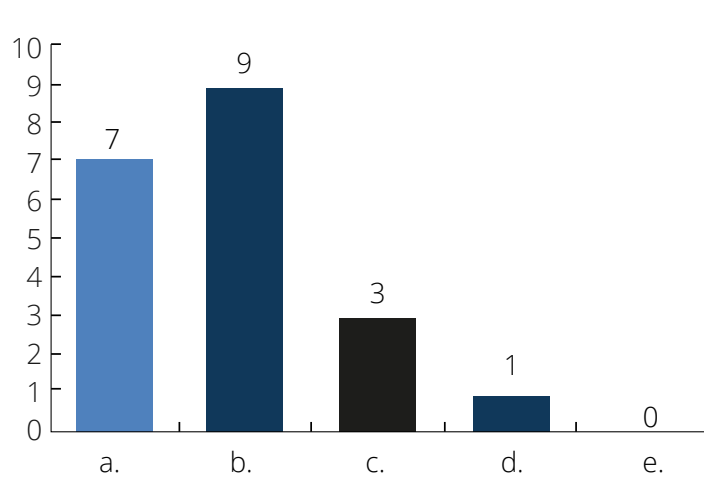

a. Siempre b. Frecuentemente

c. A veces d. Casi nunca e. Nunca

En la figura 4 se observa que la mayor parte de los docentes cumple con sus programas de asignatura y su plan docente. A una minoría de docentes no le alcanza el tiempo para poder completar su programa docente.

Con base en los resultados obtenidos, la mayor parte de los docentes frecuentemente está realizando actividades grupales y una parte indica que a veces su docente lo hace; una parte restante afirma que casi nunca su docente lo hace, lo que permite deducir que, en cuanto a los trabajos en grupo, muchos docentes implementan dinámicas en el aula.

Figura 4. El docente realizó actividades grupales. Fuente: resultados a partir de las encuestas obtenidas de los estudiantes, año 2019

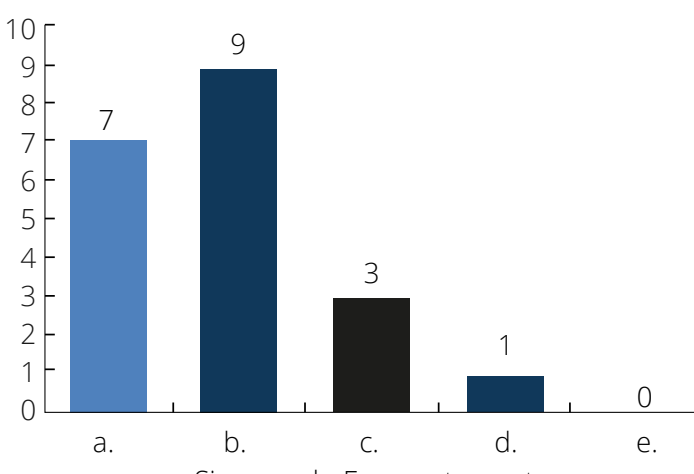

a. Siempre b. Frecuentemente

c. A veces d. Casi nunca e. Nunca

Figura 5. Planificación y ejecución de las pruebas evaluativas. Fuente: resultados a partir de las encuestas obtenidas de los estudiantes, año 2019.

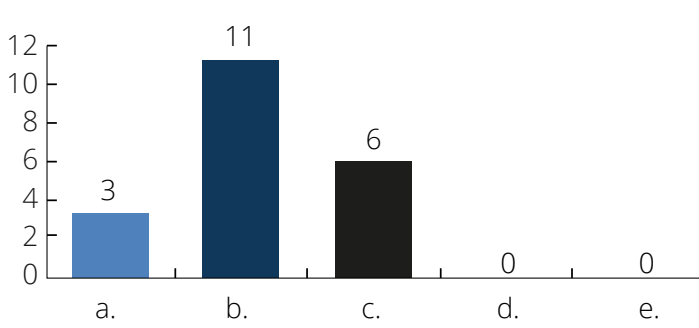

a. Siempre b. Frecuentemente

c. A veces d. Casi nunca e. Nunca

De acuerdo con los datos obtenidos, se observa que una mayoría de los docentes elabora y ejecuta sus exá- 
menes con base en los temas explicados en clase. Una cantidad menor de los docentes lo realiza a veces y un número reducido de docentes nunca elaboran las evaluaciones en función de los contenidos abordados en clases, lo que permite deducir que la mayor parte de los docentes sí consideran los temas avanzados para la elaboración de pruebas y frecuentemente planifican con anticipación las evaluaciones para que los estudiantes se puedan preparar para poder rendir de manera oportuna en las evaluaciones.

Figura 6. Claridad de las evaluaciones en las preguntas. Fuente: resultados a partir de las encuestas obtenidas de los estudiantes, año 2019.

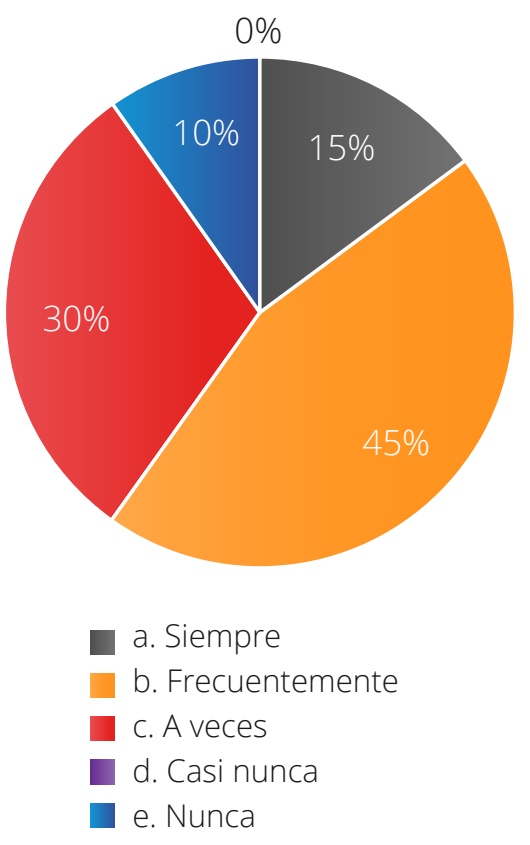

Según los resultados obtenidos, casi la mitad de los estudiantes indica que las evaluaciones son con base en lo avanzado y denotan claridad en lo que concierne a las preguntas. El resto sostuvo que no todas las evaluaciones son claras y con base en lo avanzado en clases.

Figura 7. Aclaraciones sobre las evaluaciones. Fuente: resultados a partir de las encuestas obtenidas de los estudiantes, año 2019.

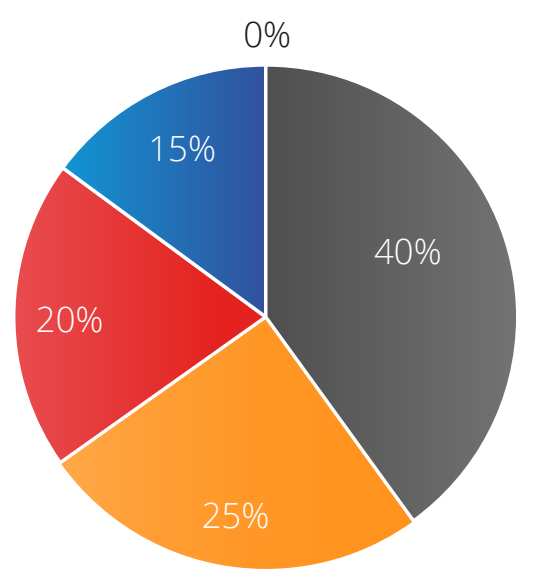

a. Siempre

b. Frecuentemente

c. A veces

d. Casi nunca

e. Nunca

Se observa una mayoría en frecuentemente y siempre los docentes aclaran las dudas que los estudiantes tienen frente a los exámenes. El resto de los estudiantes sostiene que a veces y casi nunca los docentes aclaran las dudas surgidas sobre las evaluaciones tomadas. Esto permite deducir que todavía existe una buena parte de docentes que no hace aclaraciones en las evaluaciones a los estudiantes, un factor que podría mejorarse.

Se observa frecuentemente que los docentes aceptan sugerencias para me- 
jorar en su materia. Otra parte a veces lo hace y otra nunca, aspecto que debe considerarse en el futuro, en pro de una mejora continua de la docencia.

Figura 8. Se utiliza la autoevaluación en su asignatura. Fuente: resultados a partir de las encuestas obtenidas de los estudiantes, año 2019.

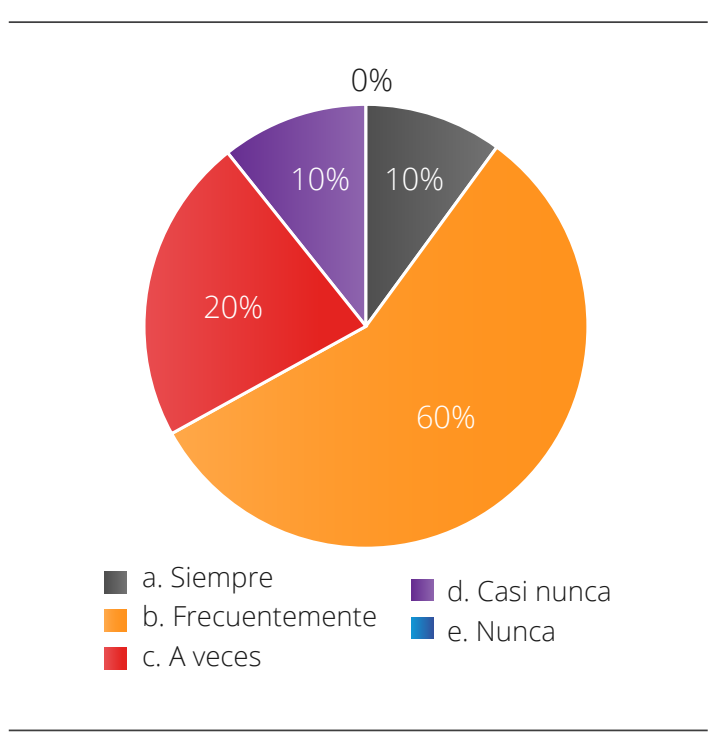

El uso de recursos didácticos se constituye un aspecto importante en la labor formativa. La información recabada muestra que la mayor parte de los docentes hace uso de recursos didácticos, un factor importante que debe considerarse. En algunos casos se observa resistencia de parte de algunos docentes hacia la utilización de recursos didácticos tecnológicos, lo que permite inferir que el uso de estos recursos tiene que ver también con su uso y falta de actualización por parte del docente.

De acuerdo con los datos recopilados, se puede observar que frecuentemente los docentes integran los valores en el aula, aunque también existe una parte de docentes no los aplican constantemente, lo cual es un factor y aprendizaje limitativo en la formación integral.

Según los resultados en cuanto a si el docente demuestra una conducta ética con sus colegas de trabajo en el aula, frecuentemente los estudiantes sostienen que sí lo hace; el 30\% afirma que siempre lo hace; el 10\% indica que a veces lo hace; el 5\% restante respondió que casi nunca lo hace, lo

Figura 9. Utilización de medios didácticos. Fuente: resultados a partir de las encuestas obtenidas de los estudiantes, año 2019.

e. Nunca

d. Casi nunca

c. A veces

b. Frecuentemente

a. Siempre
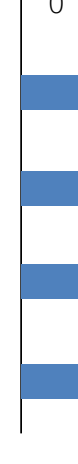

(1)

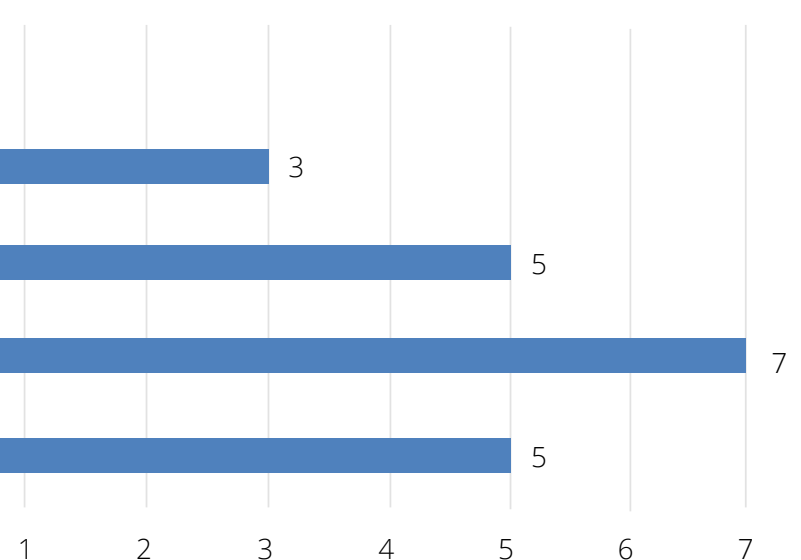

01

2

3

4

5

6


que permite deducir que existe un sesgo del trabajo docente en esta temática. Hoy en día, la dinámica es otra, cada vez hay una creciente carencia de valores.

Figura 10. Formación de valores en el aula. Fuente: resultados a partir de las encuestas obtenidas de los estudiantes, año 2019.

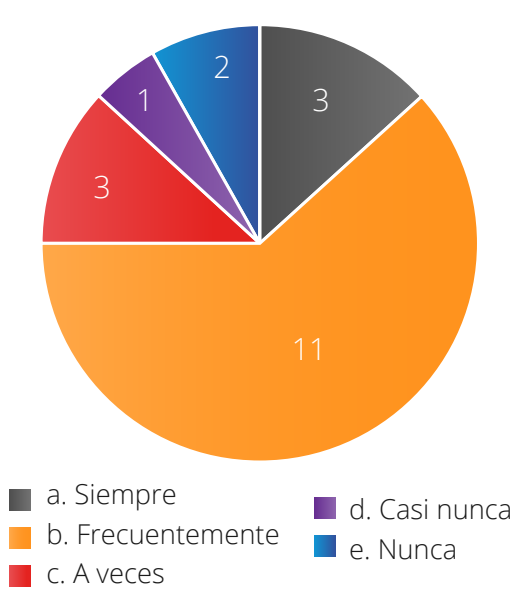

Figura 11. El docente demuestra una conducta ética con sus colegas de trabajo en el aula. Fuente: resultados a partir de las encuestas obtenidas de los estudiantes, año 2019.

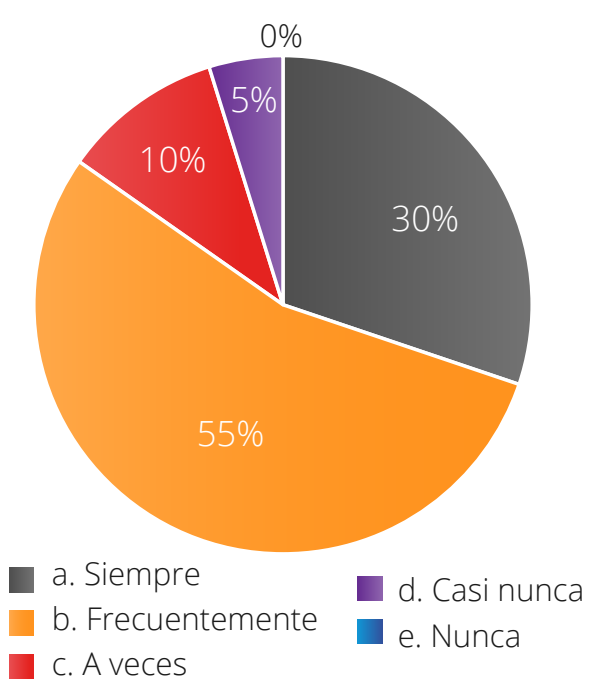

\section{Discusión}

Entre los aspectos relevantes del estudio, se logró el propósito de contar con insumos adecuados para una mejora continua de los aspectos fundamentales de la labor educativa. Dichos hallazgos resaltan la importancia del estudio.

El hecho de que los docentes no conozcan los resultados de manera oportuna de algunas evaluaciones para poder mejorar su trabajo en el aula y que en muchos casos esto se deba a factores de administración académica da lugar a la carencia de un programa de retroalimentación por parte del docente para poder mejorar su desempeño en el aula.

Por otro lado, los resultados obtenidos permitirán realizar una retroalimentación para mejorar los procesos formativos y obtener mejoras en el desempeño integral por parte del docente, y proponer nuevas formas de evaluación que sean más dinámicas y oportunas para el conocimiento de los involucrados.

El desarrollo y la madurez alcanzada por la educación superior en lo que concierne al factor humano y su importancia en la organización es un factor preponderante dentro del aula (Muñoz, 2020).

Existe una correlación con los logros y las metas, que propicia una mayor participación de los docentes en el aula (Fonseca, 2018).

Lau y Díaz (2009) plantean una permanente interacción entre los elementos 
del proceso de enseñanza-aprendizaje como forma de organización y trabajo dentro de la organización.

La didáctica de la enseñanza superior da una visión acerca de la importancia de que el docente interactúe en el aula con los estudiantes, lo que permite un mayor compromiso entre ambos actores en el proceso formativo (Escudero Muñoz, 2006; ÁlvarezRojo, 2009; Rué, 2007).

Existe una serie de preguntas de conocimiento general que coadyuvan a la obtención de datos sobre la asimilación de contenidos (Ontoria et al., 2006).

Según los resultados obtenidos en la investigación, en el aspecto de trabajo y cumplimiento del plan de asignatura, se puede deducir que los docentes que cumplieron en su totalidad su plan de clase pudieron llevar a cabo una retroalimentación permanente y la dosificación de los contenidos.

La tasación debe orientarse de manera oportuna con la finalidad de mejorar los procesos en el trabajo docente, que permita el logro de objetivos dentro de la labor formativa (Gómez-Ruiz et al., 2013).

De acuerdo con los resultados sobre la valoración del proceso de enseñanza aprendizaje según los datos obtenidos, la mayor parte de los docentes frecuentemente formulan pruebas o exámenes con preguntas claras y no ambiguas. Los restantes sostienen que existen pocas ocasiones en las que el docente formula pruebas claras, lo que permite al estudiante responder en función de lo avanzado. Según Sloep y Berlanga (2011), la complacencia de lo avanzado debe concluir con la formulación de interrogantes de las evaluaciones que vayan en la misma dirección.

La información también permitió observar que los docentes en ocasiones realizan complementación en las aclaraciones de los puntos abordados y en raras ocasiones lo hacen oportunamente.

La implementación de recursos didácticos en las labores académicas se hace necesaria en estos tiempos, lo que permite deducir que se debería implementar talleres de capacitación en la utilización de los recursos tecnológicos que se usan en las aulas. La mayoría de los docentes están motivados para desarrollar talleres de capacitación en el uso de los recursos tecnológicos. El ejercicio docente debe promover un trabajo participativo (Unesco, 2008).

Así mismo, fuera de poder implementar las TICS en los espacios de enseñanza, en la mayoría de los casos, los docentes toman como ejemplo las situaciones reales, es decir, aquellas que se encuentran en nuestro medio, para contextualizarlas y poder ser más objetivos.

Con respecto al acervo bibliográfico, se cuenta con poco material actualizado, lo que limita el acceso a una bibliografía adecuada para el desarrollo de investigación y complemento para las diferentes asignaturas regentadas. Los estudiantes también 
respondieron que algunos docentes complementan otro material como copias impresas y PDF.

En cuanto a la asistencia, frecuentemente se evidenció el cumplimiento de las actividades académicas en función de los horarios asignados. La formación en valores es un aspecto que debe considerarse, ya que estos se van perdiendo cada vez más con el paso del tiempo. Un desafío por parte de los docentes es el de trabajar más este componente en el aula.

\section{Conclusiones}

Entre las conclusiones más importantes de la investigación, en el aspecto de planificación-organización y desarrollo de la materia, los docentes cumplen frecuentemente con los planes que presentan al inicio del semestre y la organización está en función del cronograma de las actividades propuestas al comienzo de la gestión académica.

En el aspecto de evaluación, existe una tendencia a mantener los sistemas clásicos de evaluación implementados en cada asignatura, en la formulación de pruebas orales y escritas. Así mismo, existe una tendencia relativamente menor que sostiene que el sistema de evaluación denota ciertas limitaciones en cuanto a la elaboración de pruebas claras que reflejen lo abordado en las aulas. De igual manera, hay una preparación previa por parte de los docentes para poder impartir sus asignaturas en función de su designación.
En lo que se refiere al uso de medios o recursos tecnológicos, cabe mencionar que frecuentemente algunos docentes necesitan asistencia técnica para poder implementar nuevas tecnologías en el aula, lo que lleva a requerir talleres continuos de capacitación para poder mejorar el desempeño en el uso de estos recursos.

También se refleja cierta improvisación en algunas asignaturas por parte de algunos docentes, especialmente en los cursos autofinanciados que se imparten en los semestres académicos. Con respecto a la autoevaluación de las asignaturas, cabe mencionar que frecuentemente no todos los docentes realizan una valoración de su trabajo con los estudiantes, que permita una retroalimentación que busque una mejora continua de la administración académica en cada asignatura impartida en el aula.

Otro de los aspectos importantes que debe señalarse en el presente estudio y considerarse en la formación de los profesionales es el fomento del emprendimiento, debido a que anteriormente el plan curricular se orientaba a formar empleados y no emprendedores, lo que limita el espíritu emprendedor.

Se notó que muchos de los docentes no realizan una autoevaluación de su materia al final de cada semestre académico y que a veces o pocas veces la llevan a cabo en sus asignaturas para tener otra perspectiva directa de su materia dentro de su proceso de enseñanza y aprendizaje. 
Por último, algunos docentes frecuentemente fomentan los valores integrales y otros no implementan un reforzamiento sobre los valores que debe tener todo futuro profesional. Según los resultados encontrados, se concluye que algunos docentes fomentan el espíritu emprendedor en los estudiantes, lo que permite la valoración de este aspecto, ya que, anteriormente, el currículo estaba orientado a la formación de empleados. Con el rediseño curricular se pretende formar emprendedores.

\section{Referencias}

Álvarez-Rojo, V., Asensio-Muñoz, I., Clares, J., del-Frago, R., García-Lupión, B., García-Nieto, N., et al. (2009). Perfiles docentes para el espacio europeo de educación superior (EEES) en el ámbito universitario español. Relieve, 15(1). https://bit.ly/31SAQtp

Casanova, M. A. (2012). El diseño curricular como factor de calidad educativa. Revista Iberoamericana sobre Calidad, Eficacia y Cambio en Educación, 10(4), 6-20. https://bit.ly/3t1 qmUy

Escudero Muñoz, J. M. (2006). El espacio europeo de educación superior. ¿Será la hora de la renovación pedagógica de la universidad? Universidad de Murcia.

Fonseca, L. V. (2018). Diseño del proceso de Capital Humano para el Sistema de Gestión de la Calidad de la UNAH [Tesis de grado, Universidad Agraria de la Habana].

Gilio, M. \& Ibarra, M. (2005). La construcción del conocimiento ético en la formación de maestros de Ciencias de la Educación. En: Hirsh, A. (Coord.).
Ética profesional. Construcción del conocimiento interdisciplinario (pp. 345-361). Gernika.

Gómez-Ruiz, M., Rodríguez-Gómez, G. \& Ibarra-Sáiz, M. (2013). Desarrollo de las competencias básicas de los estudiantes de Educación Superior mediante la e-Evaluación orientada al aprendizaje. Relieve, 19(1). https://bit. ly/3t0o2g]

González, S., Hidalgo, N. \& Lombillo, I. (2017). La gestión del proceso de evaluación del desempeño de los docentes en la Educación Superior. VARONA, Revista Científico-Metodológica, (64), 1-13. https://www.redalyc.org/ pdf/3606/360657467003.pdf

Lau, C. \& Díaz, M. (2009). Gestión de recursos humanos en las universidades de Cuba. Gestiopolis. https:// bit.ly/3909DMY

Muñoz, D. M. (2020). La gestión del capital humano y la evaluación del desempeño docente en la Educación Superior: perspectivas y desarrollo. Revista Ciencia Universitaria, 18(2). https://bit.ly/3dL1fPs

Muñoz, E. M., Hernández-Gracia, T. J., Martínez Muñoz, B., \& Montesinos Hernández, J. (2019). Estudio de Mobbing en una institución de educación superior en Hidalgo. Revista GEON (Gestión, Organizaciones Y Negocios), 6(2), 25-36. https://doi. org/10.22579/23463910.153

Ontoria, A., Gómez, J. \& de Luque, A. (2006). Aprender con mapas mentales: una estrategia para pensar y estudiar. Narcea. 
Organización de las Naciones Unidas para la Educación, la Ciencia y la Cultura - Unesco. (2008). Estándares de competencia en TIC para docentes. https://bit.ly/3fSUEFw

Pineda Henao, A. E. (2020). La comunicación organizacional en la gestión empresarial: retos y oportunidades en el escenario digital. Revista GEON (Gestión, Organizaciones Y Negocios), 7(1), 9-25. https://doi. org/10.22579/23463910.182
Rué, J. (2007). Enseñar en la Universidad. EI EEES como reto para la Educación Superior. Narcea.

Sloep, P. \& Berlanga, A. (2011). Redes de aprendizaje, aprendizaje en red. Revista Comunicar, XIX(37), 55-64. https:// bit.ly/3cYiNZh 